\title{
Collective operation of quantum heat machines via coherence recycling, and coherence induced reversibility
}

\author{
Raam Uzdin ${ }^{*}$ \\ Fritz Haber Research Center for Molecular Dynamics, \\ Hebrew University of Jerusalem, \\ Jerusalem 9190401, Israel \\ Department of Chemistry and Biochemistry, \\ University of Maryland, \\ College Park, Maryland 20742, USA
}

(Dated:)

\begin{abstract}
Collective behavior where a set of elements interact and generate effects that are beyond the reach of the individual non interacting elements, are always of great interest in physics. Quantum collective effects that have no classical analogue are even more intriguing. In this work we show how to construct collective quantum heat machines and explore their performance boosts with respect to regular machines. Without interactions between the machines the individual units operate in a stochastic, non-quantum manner. The construction of the collective machine becomes possible by introducing two simple quantum operations: coherence extraction and coherence injection. Together these operations can harvest coherence from one engine and use it to boost the performance of a slightly different engine. For weakly driven engines we show that the collective work output scales quadratically with the number of engines rather than linearly. Eventually, the boost saturates and the scaling becomes linear. Nevertheless, even in saturation, work is still significantly boosted compared to individual operation. To study the reversibility of the collective machine we introduce the 'entropy pollution' measure. It is shown that there is a regime where the collective machine is $N$ times more reversible while producing $N$ times more work, compared to the individual operation of $N$ units. Moreover, the collective machine can even be more reversible than the most reversible unit in the collective. This high level of reversibility becomes possible due to a special symbiotic mechanism between engine pairs.
\end{abstract}

\section{INTRODUCTION}

When particles or systems interact in a special synchronized way, they can collectively accomplish remarkable tasks that are far beyond the capabilities of the sum of individual units. An electrons Cooper pair can flow in a solid without resistance while unpaired electrons cannot. In biology, symbiotic relationship refers to the case where two different species benefit from interacting with each other. Similarly, in this work we study the collective operation of quantum heat machines. The individual units are functional heat machines. The collective machine that emerges by introducing a special synchronized interaction, is also a heat machine but with superior performance. Furthermore, the synchronized interaction is based on coherence exchange between the units and is therefore inherently quantum. Since this interaction affects only coherences in the energy basis, the machines do no exchange heat or work with each other. In a certain case we show that the machines can be divided to two species that exhibit symbiotic interrelation.

A heat engine is a device that converts heat flows into useful work. Work may take different forms. In the quantum regime it may be, for example, the amplification of light (lasing medium). Alternatively a quantum power refrigerator may use microwave radiation to take away

\footnotetext{
* raam@mail.huji.ac.il
}

heat from a device and dispose it in some ambient environment. On top of the standard uses of heat machines, it has been suggested to use quantum heat machines for non classical tasks such as entanglement creation [1] and state squeezing [2].

With the growing technological abilities to control and manipulate single atoms and ions, the construction of microscopic quantum heat machines is within reach. A single atom heat engine and an NMR refrigerator have already been built [3, 4. Suggestions for realizations in several other quantum systems include quantum dots [5, 6, superconducting devices [1, 7, 9, cold bosons [10, and optomechanical systems [11-13. In particular, the rapid advancement in fabrication technology of superconducting circuits may open the road to on chip heat machines.

The study of quantum heat machines started in [14] and has become an increasingly active area of research in recent years (see 4, 11, 15,51 for a very partial list). One of the main goals in the study of quantum heat machines is to discover and understand the fundamental differences between small quantum heat machines and their macroscopic counterparts.

Surprisingly, quantum heat machines show a striking resemblance to their classical counterparts and often exhibit classical features. However, recent studies have isolated several strictly quantum features. In 52 a protocol was presented to extract work from coherences using a thermal bath without changing the energy population. In 53 it was shown that coherent quantum dynamics can lead to faster cooling rates compared to classical dynam- 
ics. In 54 it was shown that the coherence of the working fluid in the energy basis [55] gives rise to a 'thermodynamic equivalence principle' of the three main engine types: four-stroke, two-stroke, and continuous engine. In the equivalence regime these machines still have entirely different dynamics, yet their thermodynamics features are the same. This result was recently extended to the non-Markovian regime where the machine and the bath are strongly coupled [56. For other studies of coherence in quantum heat engines see [41, 57 59.

More relevant to the present work, is the identification of two distinct work extraction mechanisms [54]: a coherent (quantum) mechanism, and a stochastic (classical) mechanism. The coherent mechanism operates on coherences in the energy basis while the stochastic mechanism operates on energy populations (population inversion). Thus to observe quantum effects in power and heat as in [54, the coherent mechanism must be more pronounced than the stochastic mechanism. For this to occur it is critical that the thermal strokes be significantly shorter than the thermalization time. As a result the work stroke (unitary evolution) starts with non zero coherences and the coherence work extraction becomes important. For a study of the role of coherence in specific machines see [53, 57]. For additional studies of coherence in quantum thermodynamics see [30, 32, 36, 52, 60, 64.

One of the finding of the present work is that even if only the stochastic work extraction mechanism operates (i.e, the device is a stochastic machine), there is still a quantum resource that can be exploited for other purposes. This quantum resource is coherence. Although coherence is regenerated in each cycle in the engine by interacting with the classical field, it does not participate in the stochastic work production process. Hence, the residual coherence can be used and consumed for other purposes without affecting the performance of the engine. It is shown here that coherence obtained from one engine can be injected to a second engine, and boost its power output by activating its coherent work extraction mechanism. The present paper studies the possibilities entailed in coherence alteration processes in quantum heat machines. These processes not only give rise to new types of devices, but also have profound implications on reversibility and entropy balance in quantum heat machines. To illustrate our findings we have chosen the smallest and simplest heat engine model that can exhibit them. Nevertheless, our findings are general and can be applied to other more complex devices.

Recently, several studies considered quantum heat engines with a working fluid that contains multiple particles 65 67. In 68 a central spin is used to synchronize the other particles. These very interesting studies should be distinguished from the present study which focuses on multiple engines that operate collectively.

Section II introduces the basic unit that will be used to construct a collective machine. In section III the over-thermalization regime in quantum heat machines is briefly described. Then, in section IV we introduce the coherence extraction $(\mathrm{CE})$ and coherence injection $(\mathrm{CI})$ processes. In section $\mathrm{V}$ we quantify the amount of entropy reduction associated with CE. Next in section VI the 'entropy pollution' irreversibility measure is introduced and used to show the impact of $\mathrm{CE}$ on non collective heat machines. Section VII studies collective machine operation where $\mathrm{CE}$ and $\mathrm{CI}$ are used for coherence sharing between the engines. After studying the performance boost gained by the collective operation we give our conclusion in section VIII.

\section{THE BASIC UNIT - A TWO STROKE THREE LEVEL QUANTUM HEAT MACHINE}

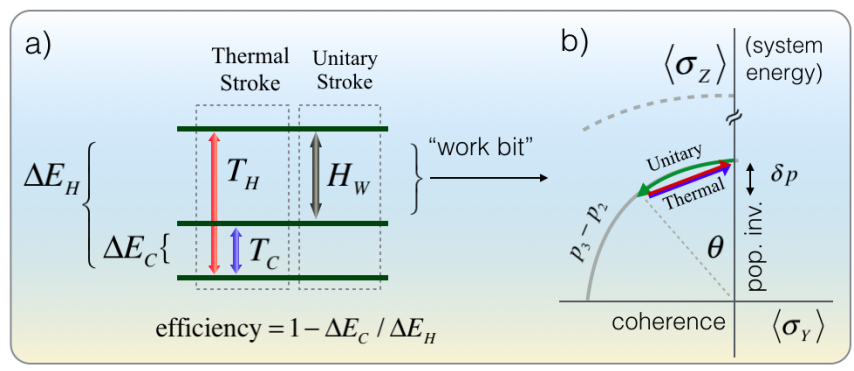

Figure 1. (a) The three-level two-stroke engine used as a basic unit for the collective machine. In the thermal stroke the hot bath operates on levels $1 \& 3$ and the cold bath on levels $1 \& 2$. The unitary stroke that follows reduces the energy of the system and extract work. (b) The engine dynamics can be visualized on the Bloch sphere of level $2 \& 3$. The $\left\langle\sigma_{y}\right\rangle$ coordinate corresponds to coherence and the change in the $\left\langle\sigma_{z}\right\rangle$ coordinate determines the change in population inversion $\delta p$ and consequently also the change in the energy of the system. The top dashed arch corresponds to the pure states shell of the Bloch sphere. The mixed state dynamics of the engine takes place inside the Bloch sphere. The maximal population inversion is determined by the previous thermal stroke (red-blue arrow). An constant radius curve with an angle $\theta$ (green) is formed by the unitary evolution of the work stroke (generated by $H_{w}$ ). In the over thermalization regime the unitary stroke generates coherence and the thermal stroke fully erases them. Thus, in the above setup there are no initial coherences in the work stroke and the device operates as a stochastic machine with no quantum effects.

The basic machine unit used in this paper is shown in Fig 1a. It is a simple two-stroke quantum engine. Strokes are time segments in which the engine undergoes different transformations [54. In the thermal stroke levels 1 and 3 are connected to a hot bath, while levels 1 and 2 are connected to a cold bath. After a period that sufficiently exceeds the thermalization time, the populations are determined by the Gibbs factors $p_{2} / p_{1}=e^{-\Delta E_{c} / T_{c}}, p_{3} / p_{1}=e^{-\Delta E_{h} / T_{h}}$. In the work stroke that follows, the baths are disconnected and a unitary operation is applied to levels 2 and 3 . Work is extracted during the unitary operation (e.g. the unitary can be a $\pi$ pulse that takes the particle to a lower energy state 
and emits an extra photon in the process). This is a twostroke quantum heat engine 39. For more information about various engine types see [54]. For a more general introduction to quantum heat machines see reviews 6972 and references therein. These reviews also cover other topics of interest in the more general and rapidly growing field of quantum thermodynamics. When the bath are disconnected (work stroke) the Hamiltonian of the engine shown in Fig. 1 is

$$
\begin{aligned}
H= & H_{0}+H_{w}(t)=\Delta E_{c}|2\rangle\left\langle 2\left|+\Delta E_{h}\right| 3\right\rangle\langle 3| \\
& +\epsilon(t) \cos \left[\left(E_{3}-E_{2}\right) t\right](|2\rangle\langle 3|+| 3\rangle\langle 2|) .
\end{aligned}
$$

Although we will use this model for illustration, our findings are more general and are not limited to this specific three-level model Hamiltonian. The interactions with the baths are described by Lindblad operators that depend on temperature.

The engine in Fig. 1 is an Otto engine. Unlike the Carnot engine, the thermal stages are isochores and not isotherms. This means that during the thermal stage the 'volume' is fixed and not the temperature. In discrete quantum heat machine a constant volume means that the energy levels are not changed in time (as they must change, in a Carnot machine). Since presently it seems that isochores are easier to implement compared to isotherms, Otto engines are the default choice in the quantum regime. The efficiency of Otto engines with uniform level compression in the work stroke [73, and also for the current engine, is $\eta=\frac{W}{Q_{h}}=1-\Delta E_{c} / \Delta E_{h}$ where $W$ is the work per cycle and $Q_{h}$ is the heat taken from the hot bath. We point out that thermodynamic bounds on efficiency refer to steady state operation and not to transients or single shot non periodic evolution. Although the efficiency in this case does not depend on the temperature, it is still limited by the Carnot efficiency $\eta=1-\Delta E_{c} / \Delta E_{h} \leq 1-T_{c} / T_{h}=\eta_{c}$. When $\Delta E_{c} / \Delta E_{h} \geq T_{c} / T_{h}$ the machine starts to work as a refrigerator rather than as an engine.

In 14 (see 74 for a more detailed analysis) it was shown that the Otto engine-refrigerator crossover point (sometimes called the Carnot point) takes place when the baths cease to generate population inversion. It is important to point out that this engine-refrigerator crossover is valid for "standard" quantum heat machines where only the baths appear in the entropy balance over one cycle. In this paper we introduce the coherence injection process. This process changes the entropy balance and therefore enables the machine to perform as an engine even without population inversion. This is related to lasing without inversion 75, but has a more general quantum thermodynamic explanation based on the notions of active and passive states as explained later on (see also Sec. III.D of [54]).

In this study the unitary is executed by a classical field (e.g., a laser light, an RF pulse, or a slow magnetic field). This is experimentally and theoretically well motivated in a wide range of physical systems in atomic physics. Some studies replace the classical field by a quantum battery (work repository) [36, 56, 60, 76, 77]. This is very interesting, and deserves further study. Nevertheless, the classical field framework is highly useful in many quantum systems where second quantization effects are not important. The classical field is in some sense a source of coherence (see, for example, 63]). However its coherence is so abundant that when the classical field approximation is valid there is no need to discuss the degradation of the classical field 63. In short, our framework is no different from that used in NMR or common laser and atomic physics setups.

\section{BLOCH SPHERE ENGINE DYNAMICS IN THE OVER-THERMALIZATION REGIME}

In the over-thermalization regime the thermal baths are connected for periods significantly longer than the thermalization time, so for all practical purposes coherences are wiped out. Consequently, the population dynamics in the unitary stroke can be fully described by stochastic means (the unitary can be replaced by a doubly stochastic operator [54]). Since the population dynamics in the Markovian thermal stroke can also be fully described by stochastic means, the quantum machine in this regime operates as a fully stochastic device. Thermodynamic observables such as work, heat and baths entropy generation, depend only on energy populations and not on energy coherences. Thus, in this regime there is little hope of observing quantum effects unless the scheme is significantly modified. The 'over' prefix in the term over-thermalization 54 is due to the fact that beyond a certain point, large thermalization rate degrades the performance of the engine, as it mitigates the coherent work extraction mechanism. This degradation due to over-thermalization has been previously observed in specific models in [19, 74, but in [54] it was understood as a generic effect. In this work we show that even in this seemingly classical-stochastic regime of overthermalization, quantum thermodynamic effects can be observed.

For work extraction, the exact details of the thermalization mechanism are not important since throughout the paper we assume over-thermalization. However for bath entropy generation accounting we do use the weak coupling result to relate the heat and the bath entropy change via $d Q=-T d S_{\text {bath }}$ where $T$ is the temperature of the bath. The weak coupling limit is widely applicable in many physical systems and constitutes the standard approach in quantum open systems 78 . In the over thermalization regime, the system is arbitrarily close to the thermal steady state when it ends the thermal stroke. The term thermal steady state refers to the state of the machine after it was connected to the baths (both of them) for a period which greatly exceeds the thermalization time. Stated differently the thermal steady state is the fixed point state of the system when it is connected to the baths. In general it is different from the periodic 
steady state of the machine which takes into account also the unitary stroke.

\section{A. Bloch sphere representation of the engine dynamics}

It is highly useful to plot the dynamics of the engine in Fig. 1a in the Bloch sphere representation. In steady state operation, the ground state population is fixed in time. The unitary never changes it, and therefore to maintain steady state periodicity, the thermal stroke cannot change it either. Thus, the whole dynamics is encapsulated in the two excited levels and can be most conveniently plotted on the Bloch sphere as shown in Fig. 1b. The $z$ axis corresponds to the expectation value of the $\sigma_{z}$ Pauli matrix and since the interaction is $\sigma_{x}$ (see (1)) the two-level "spin" will rotate in the $y z$ plane. The expectation value of $2 \sigma_{z}$ is the population inversion $p_{3}-p_{2}$ and the expectation values of $\sigma_{y}$ is the y coherence $\frac{1}{2} i\left(\rho_{23}-\rho_{32}^{*}\right)$ in the energy basis (there is no $x$ coherence in this setup). Therefore a motion along the $z$ axis is proportional to energy change, and a motion along the $\mathrm{y}$ axis is proportional to coherence change. In particular the $z$ axis itself indicates zero coherence.

In the over-thermalization regime the thermal stroke must end on the $z$ axis. Unitary evolution rotates the Bloch vector (green curve) but cannot change its radius (which is equal to the half the population inversion). The angular spread of the unitary curve depends on the interaction strength $\epsilon$, and on the stroke duration. Within the standard rotating wave approximation a $\pi$ pulse leads to a $\pi$ rotation, a $\pi / 2$ pulse leads to a $\pi / 2$ rotation, and so on. The thermal stroke generates a non unitary evolution (red-blue arrow). In steady state operation, this brings back the Bloch vector to the starting point of the unitary stroke and closes the engine cycle. In the over thermalization regime this starting point is the thermal steady state.

\section{B. Weakly driven machines $\delta \theta \ll \pi$}

In this paper we shall assume that the units are weakly driven so that $\theta \rightarrow \delta \theta \ll \pi$. Nonetheless, $\delta \theta$ is finite and not infinitesimal. Weakly driven machine don't exhaust their population inversion in each cycle and therefore, in some sense, don't reach their full potential. Nevertheless, this does not necessarily means low power since smaller changes in the engine can be completed in a shorter time.

Clearly by increasing the external driving field, more work can be extracted. However, in this work we shall assume that small $\delta \theta$ engines are given and that $\delta \theta$ cannot be increased beyond the given value. The question is what can be done to increase the work output without adjusting the drive. For a single unit this is not possible, but we show that for multiple units that operate collectively it is possible. In practice, $\delta \theta \sim \pi / 3$ is small enough to start observing the boost of the collective machine.

Weak drive may result from practical reasons. One reason may be the lack of strong enough coherent laser source in the needed frequency. Alternatively the source can be strong but off the atomic resonance. The large detuning reduced the amplitude of the Rabi oscillation (directly related to $\delta \theta$ ). Another motivation to use weak drive is to avoid excitation of other undesired mechanism and effects such as nonlinear multiphoton processes.

\section{COHERENCE EXTRACTION AND COHERENCE INJECTION}

With the exception of exact permutation, any unitary that operates on a diagonal state generates coherences in the same basis. This is especially true for unitaries that are close to the identity where this is the leading effect generated by the unitary. For diagonal states and a small time step $d t$, the change in population is proportional to $d t^{2}$ while the change in coherence is proportional to $d t$. However, as explained earlier, in the over thermalization regime this coherence is erased by the baths in the next stroke.

The idea that facilitates our main results is simple. Instead of letting the bath erase the coherence we wish to extract and store it elsewhere for further use. We impose the condition that the extraction does not change the energy population as this may lead to heat or work energy exchanges that significantly modify the thermodynamic scheme. Therefore, the coherence extraction has no energetic role only an entropic one. A simple way to implement this extraction is to swap the engine particle with a particle that has the same energy levels and the same populations but without any coherences. We call this particle coherence acceptor. This coherence extraction scheme is illustrated in Fig 2a. Since the engine operates in the stochastic regime (over thermalization), only populations matter, and this swap operation will have zero impact on the operation of the engine. All energy currents (heat and work) will remain as they were before the CE. In the weak coupling limit to the baths (that leads to the Markovian dynamics), the bath entropy generation is determined by the heat via $\Delta S_{b a t h}=-Q / T(T$ is the temperature of the bath). Therefore $\Delta S_{\text {bath }}$ is also indifferent to the presences or lack of coherences at the beginning of the thermal stroke.

To graphically describe CE two Bloch spheres are needed: one for the engine and one for the acceptor particle. As shown in Fig. 2b, at $t_{1}$, just before the thermal stroke, a swap operation starts between the engine and the acceptor (golden arrows). When it ends at $t_{2}$, the acceptor has coherence while the engine reaches the $z$ axis and looses all coherence. Since both particles have the same populations, the population inversion $(z$ coordinate) is fixed as well during the swap. As a result, the system energy does not change and the CE operation does not involve any heat or work exchange between the 

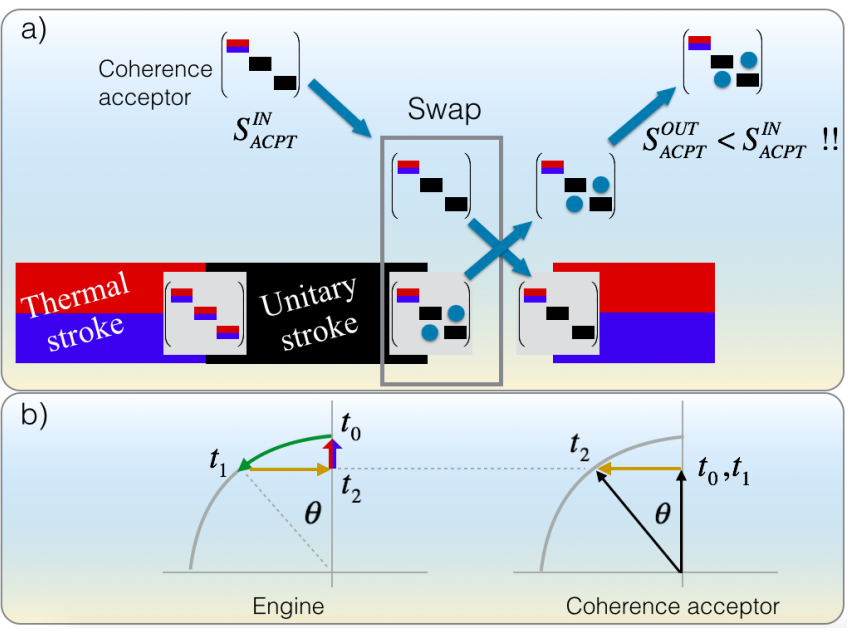

Figure 2. Introducing the coherence extraction (CE) process. (a) A density matrix illustration. The thermal stroke (bottom left of (a)) sets the engine in a diagonal state. Then, the unitary stroke generates coherences and some population changes in level $2 \& 3$. Next, the engine particle is swapped with an acceptor particle that has the same energy levels and the same population. This swap does not involve heat or work exchange. The engine state becomes diagonal but the engine performance are not affected by this. On the other hand, the gained coherence of the acceptor can be used to boost the performance of a different engine that operates with slightly different temperatures. This shows that even in the stochastic regime quantum heat engine have quantum byproducts that can be used. b) Illustration of CE in Bloch sphere representation (see text).

system and the auxiliary particle.

Later on coherence extraction will be used together with its complimentary process, coherence injection, to generate interaction between machines that lead to collective operation. However, before using $\mathrm{CE}$ for constructing a new type of quantum heat machines, it is worthwhile to discuss some immediate implications of $\mathrm{CE}$

\section{Benefits of coherence extraction}

\section{A. Cooling via $\mathbf{C E}$}

Coherence extraction adds coherence to some external particle without affecting the operation of the engine. What is this coherence good for? First, in terms of entropy, adding coherences while keeping the diagonals the same, means that the von Neumann entropy of the particle has been reduced (the state becomes purer). This is a form of cooling but without changing the average energy of the particle. Since the entropy of the auxiliary particle has been reduced and the entropy production in the baths remained the same, the total entropy production in the world (see entropy pollution later on) has been reduced. After we quantify it in Sec. VI it will become clear that this can be a dramatic effect with important consequences.

\section{B. State activation via $\mathrm{CE}$}

In terms of energy, the added coherence is also important since the state becomes more active (less passive) than it was before. Passive states are diagonal states in the energy basis whose populations are decreasing with energy 79 81. Stated differently, passive states have no coherences and no population inversion in the energy basis. All other states are termed 'active states'. The reason for the names 'active' and 'passive' is the following. It is impossible to extract energy from a passive state by a unitary transformation if the Hamiltonian is returned to its initial value after the transformation is completed (e.g. a $\pi$ pulse). On the other hand, some energy can always be extracted from active states using unitary operation until they become passive 79 .

Even if the state had some population inversion so that it was not passive to begin with, adding coherences will make it more active. To see this, consider a two-step process where in the first step permutations are used to extract the energy from the population inversion. This stage extracts work which is equal to the work extracted from the same original state but with no coherences. However, since permutations do not alter the magnitude of coherences, the state remains active. In the second step, a rotation is performed to bring the state to a diagonal form (whose energy is guaranteed to be lower). Thus the coherence extraction make the auxiliary state more active and therefore increases the energy that can be extracted from it. In some cases the acceptors need to have some population inversion, that is, they need to be active to begin with. However, one can show that with help of two $\pi$ pulses it is possible to use a passive acceptor in this case as well (without an additional energy cost).

At this point it is clear, at least qualitatively, that $\mathrm{CE}$ can cool and activate a state simultaneously. We point out that in principle, in other processes, it is possible to cool without activation and to activate without cooling. Here, however, these two good things happen simultaneously. Naturally the question that comes to mind is whether these gifts come for free, or are there some additional consumed resources? As explained earlier CE in over-thermalization does not change the heat and work flows of the engine. Nevertheless, there could be other non energetic resources. The immediate suspects for extra resources are the acceptor particles, since they have to be prepared in a specific state.

In 82 we have presented a new way of keeping track of resources in a very general setup, and showed that the acceptor particles in $\mathrm{CE}$ are not resources but separate objects that perform a different task from that of the engine. This extra task uses the coherence byproduct of the engine as fuel. Hence the cooling and activation 
are not exactly free, but are carried out without extra additional resources on top of what the engine is already consuming (heat). The curios reader is encouraged to follow the logic of resource accounting in Sec. VIII of 82 (v1). However this accounting is not needed for the collective machine analysis studied here.

Before doing the entropy balance for the $\mathrm{CE}$ process let us introduce another useful process and then do the entropy balance for both processes together.

\section{COHERENCE INJECTION}

The coherence injection (CI) process shown in Fig. 3a is the time-reversal, complementary process of coherence extraction. A 'coherence donor' particle with coherence is swapped with the engine particle that has no coherences. The donor particle and the engine particle have the same energy levels and the same energy populations. Thus, like CE, CI does not involve any energy exchange. However, in contrast to coherence extraction this process is carried out before the unitary stroke. The injected coherence allows to extract more work for the same unitary operation. This is easily understood in the Bloch picture. Consider a unitary rotation with a small angle $\delta \theta \ll \pi$. If there is population inversion but no coherence, the change in the projection on the $z$ axis (work) is $1-\cos \delta \theta=\frac{1}{2}(\delta \theta)^{2}+O\left[(\delta \theta)^{4}\right]$. On the other hand, with coherence there will be a linear term as well. If the maximal amount of coherence is injected [83. before the rotation, then the Bloch vector starts on the equator. This time the small rotation will lead to an energy change proportional to $\delta \theta$. Alternatively stated, the injected coherence enables to get larger change in the populations (z coordinate) for the same rotation $\delta \theta$.

Another use of coherence injection is to change the character of the machine without changing the temperatures. As mentioned earlier without CI the engine in Fig. 1 must satisfy $\Delta E_{c} / \Delta E_{h} \geq T_{c} / T_{h}$ in order to have population inversion and extract work. In Fig. $3 \mathrm{~b}$ we show that if coherence is injected, it is possible to extract work (have an engine) even when there is no initial population inversion. Once the Bloch vector is not pointing to the south pole, i.e. it is an active state, it is possible to apply a rotation that will decrease the energy of the system ( $z$ coordinate) and extract work by rotating the vector towards the south pole. Note that the efficiency is still given by $1-\Delta E_{c} / \Delta E_{h}$. In this particular engine CI allows do more work but this is compensated by taking more heat from the hot bath so the efficiency stays the same. As pointed out in 75, 84, coherence can be used to extract energy from a single bath. This is fully consistent with the second law when taking into account the coherence that has to be supplied to the machine.

On its own coherence injection is interesting but not very practical. In CI it is clear that coherence donors are a consumed resource that has to be accounted for. Thus, in the present paper CI is used only as part of a

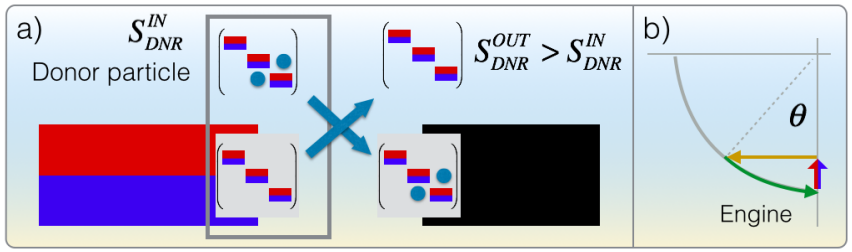

Figure 3. (a) Coherence injection (CI). CI is the time inversion of CE. Potentially it can dramatically boost performance as the unitary stroke can produce more population change if it starts with some initial coherence. Due to the complexity of preparing the coherence donor particles, this process will only be used as a part in a larger device where the donors are generated automatically by other parts in the device. In fact, one engine will be used as a donor for a different engine that will be used as an acceptor. CI can significantly extend the working regime of engines. (b) Shows an engine operating without population inversion. This engine can operate also when $T_{c}=T_{h}$ (a single bath) since the influx of coherence prevents a conflict with the second law.

larger device. In this larger device the donors are created as a byproduct of a different element in the device. This scheme is very different from other proposals that use "coherent baths" to supply the machine with coherent (non-thermal) fuel ("phasonium" 84, 85, and "squeezed baths" 86, 87]). In [53] it is assumed that coherence is somehow added to the setup. Coherence injection is a way to add this coherence without energy costs.

One may suspect that in such a closed device that has only work and thermal baths terminals, all benefits of CI and CE will vanish and the device will operate as a standard thermal machine. However we show that this is not the case and that the internal CI and CE lead to observable quantum thermodynamic signatures, and to significant performance boost.

\section{THE SECOND LAW WITH ACCEPTORS AND DONORS}

Earlier it was qualitatively shown that the engine reduces the entropy of the acceptor particles (one particle per cycle). In this section we want to quantify this effect, and show that the scaling properties of this cooling effect has a very significant consequence to the theory of heat engines and their reversibility. For this purpose we find the following two identities extremely useful:

$$
\begin{aligned}
& S\left(\rho_{2}\right)-S\left(\rho_{1}\right)=-\operatorname{tr}\left[\left(\rho_{2}-\rho_{1}\right) \ln \rho_{1}\right]-D\left(\rho_{2} \| \rho_{1}\right), \\
& S\left(\rho_{2}\right)-S\left(\rho_{1}\right)=-\operatorname{tr}\left[\left(\rho_{2}-\rho_{1}\right) \ln \rho_{2}\right]+D\left(\rho_{1} \| \rho_{2}\right),
\end{aligned}
$$

Where $S(\rho)$ is the von Neumann entropy and $D\left(\rho_{2} \| \rho_{1}\right) \triangleq$ $\operatorname{tr}\left[\rho_{2}\left(\ln \rho_{2}-\ln \rho_{1}\right)\right] \geq 0$ is the quantum relative entropy [88. Using (3) for the acceptors, and (4) for the donors we readily obtain

$$
\begin{gathered}
\Delta S_{a c p t}=-D\left(\rho_{\text {acpt }}^{\text {after }} \| \rho_{\text {acpt }}\right)=-C\left(\rho_{w}\right), \\
\Delta S_{d n r}=D\left(\rho_{d n r} \| \rho_{d n r}^{a f t e r}\right)=+C\left(\rho_{d n r}\right)
\end{gathered}
$$


where $\rho_{w}$ is the density matrix after the unitary stage and $\rho_{a c p t}, \rho_{d n r}$ are the state of the acceptor and the donor before the $\mathrm{CE}$ and $\mathrm{CI}$ take place. The coherence measure $C$ is given by $C(\rho) \triangleq D(\rho \| \operatorname{diag}(\rho)) \geq 0$. $C$ was identified in [89] as a proper coherence monotone. Furthermore, this form appeared in 61, in the context of thermodynamic transformations.

Our first main finding is that the total entropy $\Delta S_{t o t}=$ $\Delta S_{\text {baths }}+\Delta S_{a c p t}+\Delta S_{d n r}$ after one cycle of the machine is

$$
\Delta S_{t o t}=D\left(p_{w}|| p_{e q}\right),
$$

where $p_{w}$ are the populations after unitary stage and $p_{e q}$ stands for the thermal steady state population. The engine entropy does not appear in the entropy balance because in steady state the engine state is periodic so after one cycle $\Delta S_{\text {engine }}=0$. Since the relative entropy is always positive (or zero), equation (7) contains within the 2nd law. However, (7) is stronger than the second law $\Delta S_{\text {tot }} \geq 0$; the exact equality can be used to study quantitatively the entropy generation and its scaling properties. It is interesting that after including the quantum effects of $\mathrm{CE}$ and CI, the second law can be accurately described by populations only. To prove (8) we write:

$$
\begin{gathered}
\Delta S^{a c p t}+\Delta S^{d n r}= \\
{\left[S\left(\rho_{w}\right)-S\left(\rho_{a c p t}\right)\right]+\left[S\left(\rho_{e q}\right)-S\left(\rho_{d n r}\right)\right]=} \\
S\left(\rho_{e q}\right)-S\left(\rho_{a c p t}\right)=S\left(p_{e q}\right)-S\left(p_{w}\right),
\end{gathered}
$$

where we have used the fact that $\rho_{d n r}$ is related to $\rho_{w}$ via unitary transformation and therefore $S\left(\rho_{d n r}\right)=S\left(\rho_{w}\right)$. Using (8) and setting $\rho_{1}=\operatorname{diag}\left(p_{w}\right), \rho_{2}=\operatorname{diag}\left(p_{e q}\right)$ in (4):

$$
\begin{aligned}
& \Delta S^{a c p t}+\Delta S^{d n r}= \\
& -\sum_{j}\left(p_{e q, j}-p_{w, j}\right) \ln p_{e q, j}+D\left(p_{w} \| p_{e q}\right)= \\
& -\sum_{j}\left(p_{e q, j}-p_{w, j}\right)\left(-\Delta E_{j} / T_{j}+Z\right)+D\left(p_{w} \| p_{e q}\right)= \\
& Q_{h} / T_{h}+Q_{c} / T_{c}+D\left(p_{w} \| p_{e q}\right),
\end{aligned}
$$

where $Z$ is a normalization factor that comes from $p_{e q}$. Since the first two terms on the right hand side of (9) are equal to $-\Delta S_{\text {baths }}$, (9) immediately leads to (7).

To appreciate the dramatic implication of (7), we study entropy generation in engines where the unitary operation only slightly changes the populations (very small rotation) with respect to the thermal steady state. Let us denote the population change as $\delta p=p_{w}-p_{e q}$. the expression for the baths entropy is:

$$
\Delta S_{b a t h s}=-\frac{Q_{h}}{T_{h}}-\frac{Q_{c}}{T_{c}}=-\sum \delta p_{j} \ln p_{e q, j} .
$$

For small $\delta p$ the expansion of the relative entropy is:

$$
\Delta S_{t o t}=D\left(p_{w} \| p_{e q}\right)=\sum_{j=1}^{3} \frac{1}{2} \frac{\delta p_{j}^{2}}{p_{e q, j}}-\frac{1}{6} \frac{\delta p_{j}^{3}}{p_{e q, j}^{2}}+O\left(\delta p^{4}\right) .
$$

$\Delta S_{\text {baths }}$ contains a linear order in $\delta p$ but as we see from (11), $\Delta S_{t o t}$ is only quadratic in $\delta p$. This means that in coherence extraction, a remarkable cancellation of the entropy generation leading order $O(\delta p)$ (10) takes place. Thus, the cooling effect of the acceptors is not a minor effect even with respect other entropy modifying elements (baths). It is a leading order effect and as we shall see in the next sections, it can lead to new types of machines and to new reversibility limits. Another important property of $(7)$ is that it holds regardless if there is a donor or not (acceptor is necessary). However, the magnitude of $\delta p$ is affected by the donors coherence as explained earlier.

\section{ENTROPY POLLUTION AND A NEW REVERSIBLE LIMIT FOR OTTO ENGINES}

Entropy generation gives some information about reversibility. However, to a certain extent, only the limit of zero entropy generation is perfectly clear. In particular, entropy generation alone is not sufficient for comparing the reversibility of two engines. For example, one engine has $\Delta S_{t o t}=1$ and work $W=1$ and the other has $\Delta S_{\text {tot }}=1$ and $W=1000$. Clearly, the second engine is more reversible. To produce the $W=1000$ as the second engine a thousand engines (or thousand more cycles) of the first kind are needed. The total entropy production of a thousand engines of the first kind is $\Delta S_{t o t}=1000$, which is clearly less reversible compared to the second engine with $\Delta S_{t o t}=1$ for the same amount of work. This motivates us to define the entropy pollution (EP) irreversibility measure as:

$$
\mathrm{EP} \triangleq \frac{\Delta S_{t o t}}{W_{t o t}}
$$

where the quantities are calculated for a whole cycle in steady state. Note that in [90] the same measure was used to quantify the charging quality of a battery. Here, however, we use 12 to quantify the reversibility of the whole setup including all the elements. One might be tempted to suggest $\eta_{c}-\eta$ as a measure of irreversibility, but the problem is that when there are multiple baths as in the collective machine the Carnot efficiency is not uniquely defined. Depending on the bath connectivity there could be many different Carnot machines with different efficiencies. In contrast, the definition 12 is free from this ambiguity.

For classical or quantum engines without coherence injection or extraction:

$$
\begin{aligned}
\mathrm{EP}_{\text {CHEE }} & =-\frac{-\left(W-Q_{h}\right) / T_{c}+Q_{h} / T_{h}}{W} \\
& =\frac{(1-\eta) / T_{c}-1 / T_{h}}{\eta}=\frac{1}{T_{c}} \frac{\eta_{c}-\eta}{\eta} .
\end{aligned}
$$

For the Carnot efficiency the entropy pollution (13) yields zero as expected. This general expression for $\mathrm{EP}_{C H e E}$ 
shows that as long as the efficiency and temperatures are kept the same, the entropy pollution is the same. It does not matter if there are multiple units operating independently in parallel or if the value of $\delta p$ is changed. Just as an example, for the Otto engine in Fig. $1 \eta=1-$ $\frac{\Delta E_{c}}{\Delta E_{h}}$ so we verify that without CE the entropy pollution does not depend on $\delta p$ (i.e. $O\left(\delta p^{0}\right)$ ). Next we study the case of an engine with CE. Using (11) we find

$$
\mathrm{EP}_{C I C E}=\frac{D\left(p_{w} \| p_{e q}\right)}{\sum_{j=1}^{3} E_{j} \delta p_{j}}=O(\delta p) .
$$

where we used $W=\sum_{j=1}^{3} E_{j} \delta p_{j}=O(\delta p)$ and 11 . This result has a profound implication as we illustrate next. Let our goal be the production of $W_{0}$ work using one engine. This $W_{0}$ is associate with some population change per cycle $\delta p_{0}$. Now instead of doing $\delta p_{0}$ per cycle we only do $\delta p_{0} / N$ per cycle and perform $N$ time more cycles compared to the previous scenario. Clearly the same amount of work $W_{0}$ is obtained in both scenarios. Yet, in the smaller steps case, the engine operates closer to the thermal steady state $p_{e q}$. Although this is similar in spirit to a quasi-static process, it is easy to verify from (13) that without CE, the entropy pollution is not affected by this splitting to small steps. Even though the system is now always closer to the thermal steady state, the irreversibility (entropy pollution) has not decreased. In stark contrast, according to 14 with $\mathrm{CE}$ we get

$$
\mathrm{EP}_{C E}=\frac{N O\left(\delta p_{0}^{2} / N^{2}\right)}{W_{0}}=O(1 / N) .
$$

By taking the quasi-static limit $N \rightarrow \infty$ we now obtain that $\mathrm{EP}_{C E} \rightarrow 0$. This is a reversible limit as no entropy generated in the total setup (acceptors+baths+engine). At first, this result seems strange. This Otto engine produces finite work and its efficiency is less than Carnot $\left(\eta=1-E_{c} / E_{h}\right)$, and yet the entropy pollution is zero, i.e. it is reversible. This seems odd as the Carnot's theorem states that two-bath reversible machines must operate at Carnot efficiency. Indeed, without CE the second law permits only one type of reversible machine - the Carnot machine. However, the inclusion of $C E$ changes the entropy balance and enables the existence of other types of reversible machines whose efficiencies are lower than that of Carnot. In fact, when expanding the acceptors entropy for small $\delta p$ one finds that the bound predicted by the second law $\Delta S_{\text {tot }}=\Delta S_{\text {bath }}+\Delta S_{\text {acpt }}=0$ leads in the limit $\delta p \rightarrow 0$ to the observed efficiency $1-\Delta E_{c} / \Delta E_{h}$ and not to the Carnot efficiency. Once again we have full consistency with the second law.

Note that this reversible limit does not have to be slow. Instead of doing $N$ times more cycles, it is possible to have $N$ engines working in parallel for the original number of cycles. Other aspects of reversibility will be discussed in a different publication.

In the $\delta p \rightarrow 0$ limit entropy is not generated. It is simply redistributed between the baths and the acceptors. In

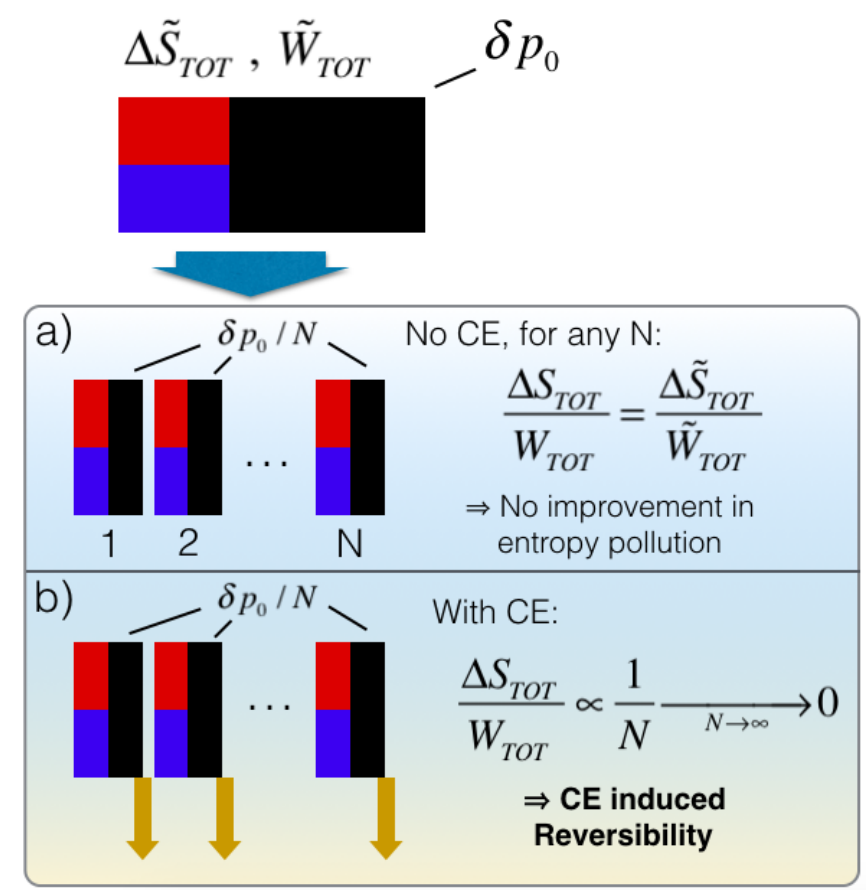

Figure 4. (Top) Splitting the work done in one cycle $\tilde{W}_{t o t}$ into $N$ many cycles, makes the system stay closer to the thermal steady state. However, as shown in 13 the entropy pollution irreversibility measure is not reduced or affected by this splitting (see (a)). In contrast, when CE is added to the scheme (see (b)), the entropy pollution goes to zero in the limit of large $N$. This enables to achieve reversibility even though the efficiency is lower than the Carnot efficiency. As explained in the text this is fully consistent with the second law.

the scenario studied above the heat and work flows were not affected by the coherence extraction. To avoid unnecessary entropy generation coherence was stored in the acceptors that act as coherence repositories. Although this is interesting from an informational point of view, our main goal in this paper is to show new ways of harnessing coherence to boost performance. Our approach to achieve this performance boost is to construct a collective operation scheme as discussed in the next section.

\section{COLLECTIVE COHERENT ENGINES}

As we have shown, $\mathrm{CE}$ in quantum heat engines reduces the entropy production but it does not affect the power performance of the engine. It enables other tasks like cooling and activation for free, but the main function of the engine, power production, does not benefit from the free coherence production. Now we present a scheme where $\mathrm{CE}$ and $\mathrm{CI}$ are used to simultaneously boost power (not efficiency) and reversibility. This scheme has another very appealing property; it does not use any external acceptor and/or donor particles as before. It is based solely on coherence sharing between engines. When two 
engines interact one engine perform as donor and the other as an acceptor.

Earlier we introduced the coherence injection process. Despite the potential power boost it offers, the preparation of a stream of coherent donor particles makes the scheme less appealing. Fortunately, as shown earlier, engines produce coherence that can be harvested and used without any energetic cost. It is natural, then, to use one engine as a donor and pass its coherence to a second engine that will serve as an acceptor. The first engine experiences coherence extraction and the second engine experiences coherence injection. The second engine gets a power boost by starting the unitary with some coherences as explained earlier and in [54]. The total power will be the power obtained from the two engines (the first stage also produce power). This process can be repeated for multiple stages until the is no more coherence to harvest from the last stage. In what follows we study two different regimes and compare to individual operation without coherence sharing.

\section{A. Quadratic power boost for collective machines}

Our collective coherent engine scheme is shown in Fig. 4a. The first engine donates its coherence to the second stage engine. The temperatures of the second engine baths are chosen so that resulting thermal steady constitutes an acceptor for the first engine. It is a perfect synergy where one stage prepares what the other stage needs while all units produce work at the same time. In addition, later on in the cycle the second stage engine donates its coherence to a third-stage engine, and so on. Each donor-acceptor interaction between the engines is carried out by a full swap of the two engine particles. Thus only engine particles are participating in this scheme and there are no external donor-acceptor particles. The question of CI extra resources is irrelevant in this device.

Due to this process of coherence transfer, each engine continues the unitary evolution where the previous stage has stopped (green line in Fig 4a and 4b). Thus, instead of many copies of $\delta \theta$ rotations as happens without CECI, the whole system performs $\delta \theta$ unitary as shown in Fig 4b. It is like an assembly line where each engine does its part. We denote by $\Omega=N d \theta$ the collective angle. For initial population inversion $d p_{0}$ the total change in the population inversion is $\delta p=d p_{0}(1-\cos \Omega)=2 d p_{0} \sin ^{2} \frac{\Omega}{2}$ and therefore the work of the collective device is

$$
W_{\text {coll. }}=\left(E_{h}-E_{c}\right) d p_{0} \sin ^{2} \frac{\Omega}{2} .
$$

Next, we wish to compare the collective operation to the operation of $N$ independent units with the same $\delta \theta$. Now that there is no $\mathrm{CE}$ and $\mathrm{CI}$ there is no need for using different temperatures for different engines. Nevertheless, for the fairness of comparison, we do not allow the standalone units to be connected to any cold and hot temperatures. The temperatures must be chosen from those available to the collective machine. This principle will be used in other comparisons later on.

We choose the unit that produces the maximal amount of work when working in a standalone mode, and set the other $N-1$ units to work with the same temperatures (N independent copies of the same engine-bath setup). The engine that produces the maximal amount of work as a standalone unit is the first one, because it has the highest initial population inversion. Thus the total power in the standalone work optimized (SWO) case is

$$
W_{N, \text { swo }}=N\left(E_{h}-E_{c}\right) d p_{0} \sin ^{2} \frac{\Omega / N}{2} .
$$

The work boost for $\Omega \leq \pi$ :

$$
\frac{W_{\text {coll. }}}{W_{N, \text { swo }}}=\frac{\sin ^{2} \frac{\Omega}{2}}{N \sin ^{2} \frac{\Omega / N}{2}} \geq \frac{\sin ^{2} \frac{\Omega}{2}}{\left(\frac{\Omega}{2}\right)^{2}} N \geq \frac{4}{\pi^{2}} N .
$$

When $\Omega \ll \pi$ the collective work boost is simply $N$ (the coefficient is unity and not $4 / \pi^{2}$ ). Alternatively stated, while in the standalone case the work for a fixed $d \theta$ scales like the number of units $N$, in the collective case the work scales like $N^{2}$.

We point out that in the performance comparison studied in this paper the 'unitary strength', $\delta \theta$, of each units is fixed. We do not compare machines with different values of $\delta \theta$. Instead, we ask the following question: given that $N$ machine with fixed $\delta \theta$ are available, is it possible to boost their performance by making them work collectively?

\section{B. Saturation and boosted linear scaling of collective machines}

The expression (16) for the collective work is bounded and reach its peak value at $\Omega=\pi$. On the other hand, the expression for the non interaction SWO units always scales like N. Does this means that for some large enough value of $N$ the collective machine will produce less power than $N$ SWO machine? not quite. Despite the saturation, collective operation still give rise to significant power boost.

Let $M=\pi / \delta \theta$ be the number of units needed for a $\Omega=\pi$ collective rotation. We have at our disposal $N \gg M$ units, each of strength $\delta \theta$ (which is fixed and $N$ independent). There is no point in letting more than $M$ units interact together. For example $2 M$ units operating collectively will generate a $2 \pi$ rotation that amounts to zero work. Hence, we divide the units into sets of $M$ units (one set may contain less than $M$ units). Each set is operated as a collective machine. For the complete sets it holds that $\Omega=\pi$, so according to 16$)$ their power amounts to $\left(E_{h}-E_{c}\right) d p_{0}\lfloor N / M\rfloor$ where $[\cdot]:=$ Floor $(\cdot)$. Adding the power from the single incomplete set we get

$$
\begin{aligned}
& W_{\text {coll }, N>M}= \\
& \left(E_{h}-E_{c}\right) d p_{0}\left\{\lfloor N / M\rfloor+\sin ^{2}(\operatorname{Rem}(N, M) \delta \theta / 2)\right\}
\end{aligned}
$$




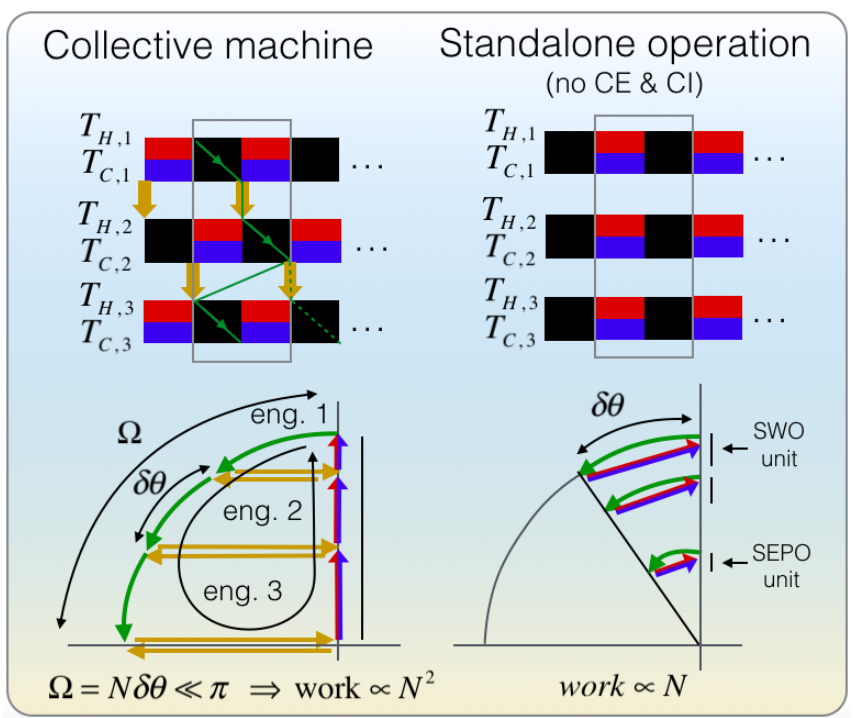

Figure 5. (left) In our collective heat machine scheme coherence is extracted from one machine and injected to the other. The resulting "collective unitary" of angle $\Omega$ produces more than $N$ times more work compared to the standalone operation (right). The work boost is $N$ even when comparing to $\mathrm{N}$ standalone units that produce the maximal amount of work (SWO units). This means that for a fixed $\delta \theta$ the collective work scales quadratically with the number of units rather than linearly as in the standalone operation.

where Rem is the remainder. For large $N$ and small $\delta \theta$ $(\delta \theta \leq \sim \pi / 5$ or $M \geq \sim 5)$ :

$$
\frac{W_{\text {coll }, N \gg M}}{W_{1, S W O}} \simeq \frac{N / M}{\delta \theta^{2} / 4}=\frac{4 M}{\pi^{2}} N
$$

Comparing this result to the SWO scaling $\frac{W_{N, S W O}}{W_{1, S W O}}=N$ we observe that there is a $\frac{4 M}{\pi^{2}}$ power boost even though the behavior is no longer quadratic for $N \geq M$. This is illustrated in Fig. 6 for $M=20$. The graph aim to show the $N \geq M$ behavior. The red dot show the actual power of the collective machine as give by expression (19). Initially the power grows quadratically (blue curve). After that the power follows the boosted linear scaling (green curve) given by (20). For comparison, the orange curve shows the linear power growth of non interacting units. For smaller $\delta \theta$ the quadratic scaling regime is larger.

\section{Extraordinary reversibility via symbiotic relations at $\Omega=\pi$ collective angle}

In this section we study the intriguing case of $N \delta \theta=\pi$. In terms of work boost we simply need to set $\Omega=\pi$ in (18). However, in this section we are interested in the entropy pollution of the collective device. Surprisingly, we find that the collective machine can be more reversible than the most reversible unit in the collective. Moreover, in this machine work yield boost and reversibility boost

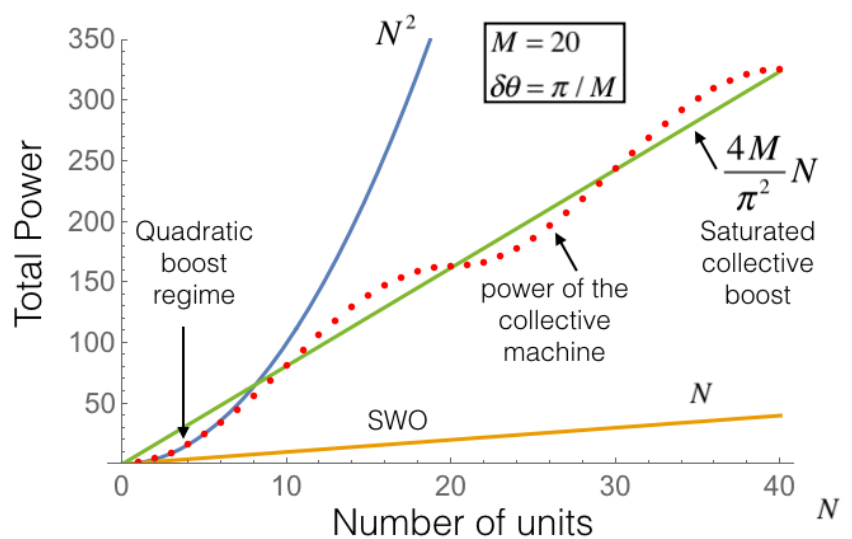

Figure 6. When the number of units $N$ exceeds the number of units needed to complete a $\pi$ rotation in the Bloch sphere $M$, the boost changes from quadratic (blue) to linear (green). Nevertheless the linear scaling of the collective machine is still boosted by a factor $4 M / \pi^{2}$ (for $M \geq \sim 5$ ) compared to the standard linear scaling $N$ (orange) of the non interacting SWO units. Depending on $\delta \theta$, the quadratic regime can be larger.

are not in conflict. Both of them increase with the number of units. This is due to a unique symbiotic mechanism that takes place in the $\Omega=\pi$ collective machine.

In the $\Omega \ll \pi$ case all units are close to the north pole, so the entropy pollution of the collective machine is very similar to that of a standalone machine with temperature that corresponds to maximal population inversion (north pole of the Bloch vector).

In addition, in the $\Omega<\pi$ collective machines there is some residual unused coherence since the Bloch vector does not return to the $z$ axis at the end of the collective unitary ( $\Omega$ rotation). Since there is no external acceptor to store this coherence it is being erased by the baths. Consequently, the bath entropy generation is larger compared to the case where coherence is stored.

For a fair performance evaluation of the $\Omega=\pi$ engine, it is important to take the right comparison reference. We shall make two different comparisons. First we compare the $\Omega=\pi$ case to the standalone work optimized case studied in the previous section. Next we shall remove the work optimization constraint and compare to the most reversible unit in the collective. First we start with the scaling of the entropy pollution in the $\Omega=\pi$ case.

\section{Entropy pollution of the $\Omega=\pi$ machine}

In our collective scheme all units but the last one, have acceptors (the next stage engine) that remove the remaining coherence after each engine complete its unitary stroke. Hence, in the general case, the entropy generation in all units but the last can be evaluated using (7). However, when $\Omega=\pi$ the last machine ends at the south pole with no coherences so there is nothing to extract. 


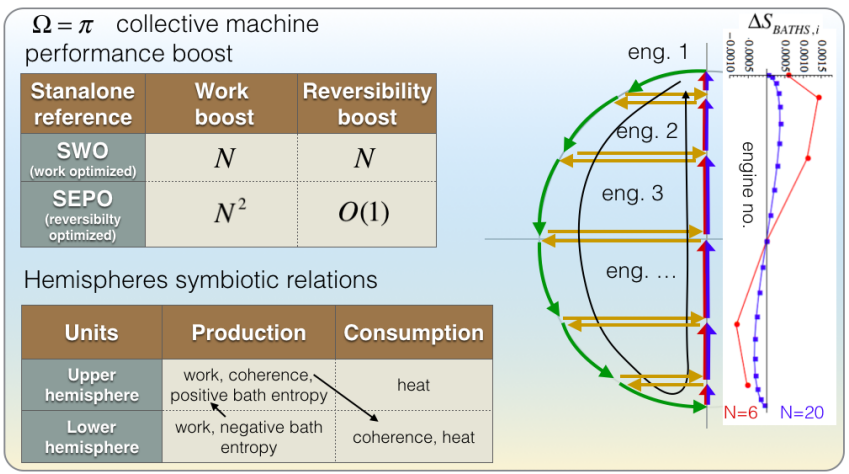

Figure 7 . The $\Omega=\pi$ collective machine shows a remarkable symbiotic interaction between units in the upper and lower hemisphere. The coherence the lower units require in order to operate as engines (they have no population inversion) is supplied by the upper units. In return the lower units produce negative bath entropy that in the limit of large $N$ compensates for the positive entropy generation of the upper units. The entropy generation in the baths of each units is shown in the rightmost graph. In the limit of large $N$ the graph becomes antisymmetric and the total entropy production goes to zero. In this limit the $\Omega=\pi$ is reversible.

Thus, in the $\Omega=\pi$ case we can safely use $(7)$ for all engines including the last one, and get that the total entropy production is

$$
\begin{aligned}
\Delta S_{\text {tot }} & =\Delta S_{\text {baths }}^{\Omega=\pi}=\sum_{i=1}^{N} D\left(p_{\text {eq }}^{(i+1)} \| p_{\text {eq }}^{(i)}\right), \\
p_{\text {eq }}^{(i)} & =\left\{1, e^{-\frac{\Delta E_{c}}{T_{c, i}}}, e^{-\frac{\Delta E_{h}}{T_{h, i}}}\right\} /\left(1+e^{-\frac{\Delta E_{c}}{T_{c, i}}}+e^{-\frac{\Delta E_{h}}{T_{h, i}}}\right),
\end{aligned}
$$

where we used $p_{e q}^{(i+1)}=p_{w}^{(i)}$. This $p_{e q}^{(i+1)}=p_{w}^{(i)}$ condition determines the temperatures of the $i+1$ baths - it is the basic population matching in CE and CI that assures that there are no energy exchanges between the units. In addition, $\Delta S_{\text {tot }}=\Delta S_{\text {baths }}$ follows from the fact that in this system there are no external particles so in a periodic process the total entropy of the elements can only be modified in the baths. For, small population change $\delta p$ associated with small $\delta \theta$, we can write: $D\left(p_{e q}^{(i+1)} \| p_{e q}^{(i)}\right)=\frac{1}{2} \sum_{j=2}^{3} \frac{\delta p_{i}^{2}}{p_{e q}^{(i)}(j)}$ and $\delta p_{i}=d p_{0} \sin \theta_{i} \delta \theta$, where $j$ is a level index and $i$ is an engine index. Using $d \theta=\pi / N$ we have $\Delta S_{\text {baths }}=\Delta S_{\text {tot }}=$ $\sum_{i=1}^{N} \frac{1}{2} \sum_{j=2}^{3} \frac{\delta p_{i}^{2}}{p_{e q}^{(i)}(j)}=\sum_{i=1}^{N} \frac{1}{2} \sum_{j=2}^{3} \frac{d p_{0}^{2} \sin ^{2} \theta_{i} \delta \theta \frac{\pi}{N}}{p_{e q}^{(i)}(j)}$. Since $p_{e q}^{(i)}(j)$ is a function of $\theta_{i}$ we get $\Delta S_{\text {baths }}=\Delta S_{\text {tot }}=$ $\frac{1}{N} \sum_{i} F\left(\theta_{i}\right) \delta \theta$. The sum can be approximated by an integral for large N. Since the integral does not depend on $\mathrm{N}$ we get that $\Delta S_{t o t}^{\Omega=\pi} \propto 1 / N$. The total work in this case is $d p_{0}\left(E_{h}-E_{c}\right)$ regardless of $N$, so finally, the entropy pollution scaling is:

$$
E P_{\Omega=\pi} \propto 1 / N
$$

\section{Comparison to the standalone work optimized case}

We saw that if the $\mathrm{N}$ units that constitute the collective engine, work independently and the temperatures are chosen to maximize the work, their total work is $N$ times weaker compared to the collective machine (see (18)). One might suspect that this enhancement is accompanied by degradation of reversibility, i.e. in an increased entropy pollution. In the standalone work optimized case the entropy pollution is the same for all units. It is given by (13) with the temperature and Carnot efficiency that fits the north pole of the Bloch sphere. The exact expression is not important. What matters is that $E P_{\text {swo }}$ does not depend on the number of units $N$. Using (23) we immediately get

$$
\frac{E P_{\Omega=\pi}}{E P_{s w o}}=\frac{O(1 / N)}{O\left(N^{0}\right)}=O(1 / N)
$$

The conclusion is that the $\Omega=\pi$ collective machine produce $N$ times more work, and at the same time it is $N$ times more reversible compared to the standalone work optimized case. In addition this shows that even though there are no external auxiliary particles, CE and CI are still highly useful as they can increase work yield and reversibility.

Note that the comparison above is very strict. If the coherence exchange is not performed but the temperature of each unit is the same as in the collective machine, then all the lower hemisphere units will turn into refrigerators and consume work instead of producing work. As a results, the total work output in this 'no CI CE' case becomes zero for large $N$ (the cancellation is not perfect for small values of $N$ ). Of course in such a case there is little motivation to use units that do not produce work. This is why we chose to compare it to the power-maximized standalone case. Next, we compare it to the standalone EP optimized case.

\section{Comparison to the most reversible unit}

If we look on the individual units operating at different temperatures needed for the collective machine, the unit with the lowest entropy pollution is unit number $\lceil N / 2\rceil:=\operatorname{Ceiling}(N / 2)$, the one that starts just above the equator 91. This unit has the lowest entropy pollution since for large $N$ its Carnot efficiency approaches the actual efficiency $\eta_{c}(\lceil N / 2\rceil) \rightarrow \eta=1-\Delta E_{c} / \Delta E_{h}$ (see (13)). Now we wish to compare the collective machine to the most reversible unit (in standalone operation) in the collective. Following the same logic as in the SWO case, $N$ copies of this most reversible unit are chosen as a comparison reference. We denote this reference by SEPO for 'standalone EP optimized'. We once again point out that for the fairness of comparison, we are only allowed to use bath temperatures that are available to the collective machine. It is simple to show that this time the 
ratio between the work of the collective $\Omega=\pi$ machine and the SEPO scales like $N^{2}$. However, when comparing the entropy pollution of the two scenarios one finds something surprising.

After some algebra one can show that the entropy pollution of the SEPO scales like $1 / N$, just like the $\Omega=\pi$ collective entropy pollution (23). In Fig. 8 the ratio $\mathrm{EP}_{\text {coll. }} / \mathrm{EP}_{\text {sepo }}$ is plotted for different minimal temperatures. As expected from the scaling arguments above, the ratio saturates for large $N$. The most striking feature in this figure is that the collective machine can be more reversible (less polluting) than the most reversible unit (the plotted ratio is smaller than one). The second feature that stands out in Fig. 8 is the dependence on the parity of units number. This parity dependence is not coming from $\mathrm{EP}_{\text {coll. }}$ but from the SEPO reference. For even $N$ the most reversible machine is at an angle $\frac{\pi}{N}$ from the equator while for odd $N$ the angle is $\frac{\pi}{2 N}$. For large $N$ this means a factor of two in the $\eta_{c}-\eta$ factor that appears in 13 . The figure shows that when the cold temperature is comparable (or higher as we see numerically) to the cold gap, the collective machine is more reversible than the SEPO regardless of parity. When the low temperature is below the cold gap we observe that only the even $N$ values lead to superior reversibility of the collective machine.

Of course this small improvement shown in Fig. 8 is not a performance boost. The performance boost is in the power as explained in the beginning of this section. Nevertheless, it is remarkable that the collective machine can be more reversible than the most reversible unit even by a little.

The two comparisons shown in the upper table in Fig. 7 , can be combined by considering the figure of merit $W /$ EP that can be understood as work multiplied by reversibility measures $1 / \mathrm{EP}$. Repeating the same argument as before it is easy to verify that the $W / \mathrm{EP}$ measure of the $\Omega=\pi$ machine is $N^{2}$ better compared to both SWO and SEPO standalone operation.

In what follows we present a different point of view that unravels the interesting dynamics that leads to high reversibility and high work yield in the $\Omega=\pi$ collective machine. The rightmost graph in Fig. 7 shows the bath entropy generation $\Delta S_{\text {baths }, i}=-Q_{h}^{(i)} / T_{h}^{(i)}-Q_{c}^{(i)} / T_{c}^{(i)}$ of each engine (red circles $N=6$, blue squares $N=$ $20)$. In the lower hemisphere there is a negative entropy production. Clearly, from (9) this becomes possible only if there is CI. The source of the injected coherence are the engines in the upper hemisphere. As the number of engines increases the graph becomes more antisymmetric and the total sum of entropy generation in the various baths becomes closer to zero. The reason for the almost exact, leading order, cancellation of the $i$ baths entropy generation with that of $N+1-i$ baths (antisymmetry), can be understood in the following way. Equation (5)-(7) lead to

$$
\Delta S_{b a t h s, i}=C\left(\rho_{d n r, i}\right)-C\left(\rho_{w, i}\right)+D\left(p_{w, i} \| p_{e q, i}\right)
$$

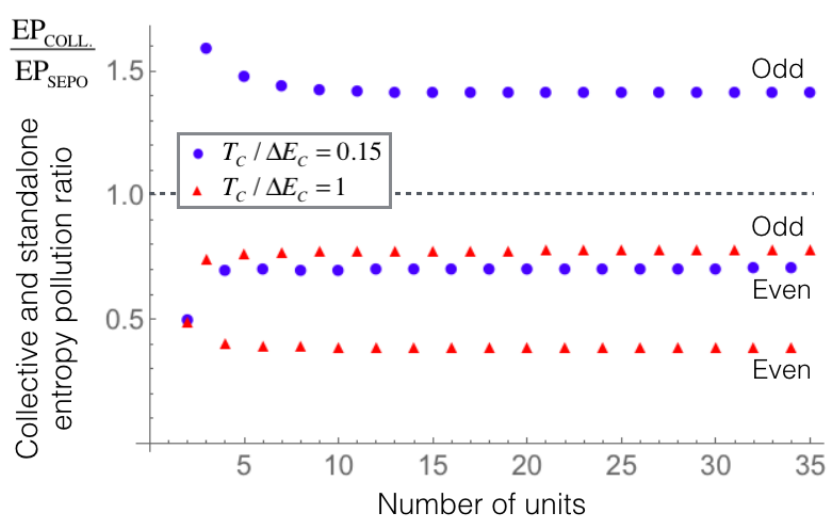

Figure 8. Comparing the entropy pollution of the collective machine to its most reversible element in standalone units (SEPO). The graph shows that the reversible collective machine can be more reversible than the most reversible unit that composes it (pollution ratio smaller than one). The parity dependence is explained in the text. The collective is not always more reversible as shown by the blue circles that correspond to a collective machine with colder baths.

The last term scales like $1 / N^{2}$, so the leading term is the coherence difference that scales like $1 / N$. Since up to level permutation $\rho_{d n r, i}$ is equal to $\rho_{w, N+1-i}$ it follows that $C\left(\rho_{d n r, i}\right)=C\left(\rho_{w, N+1-i}\right)$ and therefore

$$
\begin{aligned}
\Delta S_{\text {baths }, i}+\Delta S_{\text {baths }, N+1-i} & =D\left(p_{w, i} \| p_{e q, i}\right) \\
& +D\left(p_{w, N+1-i} \| p_{e q, N+1-i}\right) \\
& =O\left(1 / N^{2}\right) .
\end{aligned}
$$

This pairwise cancellation of the leading order, $1 / N$, in the bath entropy generation, enables the reversible limit of the $\Omega=\pi$ machine. After providing the mathematical explanation for this pairwise cancellation, it instructive to examine the unique physical circumstances that enables this result. The pairwise cancellation is a manifestation of a very interesting symbiotic relation between the engines in the upper hemisphere and the engines in the lower hemisphere. The upper units and lower units operate in a very different manner. The upper ones produce work and coherence. The engines in the lower hemisphere also produce work but since they do not have population inversion, they must use coherence in order to produce work (see Fig. 3b). The coherence they need comes from the engines in the upper hemisphere. This shows what the lower hemisphere units gain from the upper ones. What about the other direction? Do the upper ones get something in return from the lower ones? The answer is yes. As explained earlier, and as shown in the graph in Fig. 7), due to the coherence injection the engines in the lower hemisphere have negative bath entropy generation. Equation 24 implies that for large $N$ this negative contribution compensates the positive bath entropy generation of the upper hemisphere engine. In summary, the upper units 'sponsor' the lower units, while the lower units clean up after the upper ones (see lower table in 
Fig. 7). To the best of our knowledge this is the first example of symbiotic relations between different 'species' of machines in thermodynamics.

If $N$ is large enough this machine operates as a refrigerator when the order of the swap operations is reversed. With some modifications that will be described elsewhere, the reverse operation can be improved.

Note that there is no Carnot theorem for multiple baths. There are efficiency limits but for the same multiple baths there are different reversible machines that operate at different efficiencies. Only in the two bath case the reversibility constraint is sufficient to fix the efficiency. Hence, the $\Omega=\pi$ heat engine has a reversible limit with efficiency $\eta=1-\Delta E_{c} / \Delta E_{h}$ that is not fixed by the baths temperatures.

\section{CONCLUDING REMARKS}

In this work we have introduced two quantum thermodynamics operations - coherence extraction (CE) and coherence injection (CI). The first (CE) enables to reduce the entropy pollution while the latter can boost performance and extend the parameter regime in which the machine operates as an engine. By allowing heat machines to interact with each other according to these two interactions, we studied what is, to the best our knowledge, the first example of coherent collective heat machine. For certain parameters the work in this device scales quadratically with the number of composing engines and on top of the work boost it can also be more reversible compared to standalone operation. In addition, the collective machines constructed here make use of coherence extraction and injection without external coherence storage. The terminals of the collective machine are just thermal baths and a classical driving field (work repository). Nevertheless the internal coherence manipulation enable to extract more energy from the heat baths compared to individual standalone operation.

The harvesting of coherence has a significant impact on the role of the second law in heat machine operation, as less information is erased in each cycle. This is neatly expressed in a simple relative entropy expression for the total entropy production. The relative entropy scaling shows that the information salvaged in the coherence can be significant enough to enable new reversible limits for otherwise irreversible machines.

For a standard quantum machine, strong thermalization destroys coherence and turns the device into a stochastic thermal machine. The present study breaks this seemingly necessary connection between full thermalization and stochastic-incoherent operation. In the collective operation mode, full thermalization and coherence effects coexist.

The use of full swap for CE and CI was chosen to avoid entanglement generation between particles as it may increase the entropy of the reduced systems and increase the entropy pollution. Nonetheless, it is possible that entanglement created by partial swap can be put to use in some variation of the engines studied here.

$\mathrm{CE}$ and CI give rise to new types of quantum thermodynamic devices. Experimental realizations of the ideas presented here call for techniques developed for quantum computation in order to implement the swap gates. In addition, open system techniques are needed to generate the thermalization stroke. Although these techniques are not yet perfectly integrated together at the moment, both are feasible with present day technology. In particular, it seems likely that the rapidly growing maturity of superconducting circuits technology will play a major role in quantum thermodynamics devices. In the context of the present setup, superconducting technology is well suited for fabricating sets of qubits that interact with their neighbors as well as with other baths [7].

\section{ACKNOWLEDGMENTS}

Part of this work was supported by the COST Action MP1209 'Thermodynamics in the quantum regime'. The support and warm hospitality of the Walmsley group, and the physics department in Oxford University is greatly acknowledged. The author cordially thanks the support the Kenneth Lindsay trust fund. Finally, the author is in debt to Prof. Ronnie Kosloff for his extraordinary support over the years.
[1] J. B. Brask, G. Haack, N. Brunner, and M. Huber, New Journal of Physics 17, 113029 (2015).

[2] A. Zagoskin, E. Il'ichev, and F. Nori, Physical Review A 85, 063811 (2012).

[3] J. Roßnagel, S. T. Dawkins, K. N. Tolazzi, O. Abah, E. Lutz, F. Schmidt-Kaler, and K. Singer, Science 352, 325 (2016).

[4] J. Baugh, O. Moussa, C. A. Ryan, A. Nayak, and R. Laflamme, Nature 438, 470 (2005).

[5] D. Venturelli, R. Fazio, and V. Giovannetti, Physical review letters 110, 256801 (2013).

[6] C. Bergenfeldt, P. Samuelsson, B. Sothmann, C. Flindt, and M. Büttiker, Physical review letters 112, 076803 (2014).

[7] A. Niskanen, Y. Nakamura, and J. Pekola, Physical Review B 76, 174523 (2007).

[8] M. Campisi, J. Pekola, and R. Fazio, New Journal of Physics 17, 035012 (2015).

[9] J. P. Pekola, Nature Physics 11, 118 (2015).

[10] O. Fialko and D. Hallwood, Physical review letters 108, 085303 (2012).

[11] A. Mari and J. Eisert, Phys. Rev. Lett. 108, 120602 (2012).

[12] K. Zhang, F. Bariani, and P. Meystre, Physical review 
letters 112, 150602 (2014).

[13] A. Dechant, N. Kiesel, and E. Lutz, Physical review letters 114, 183602 (2015).

[14] H. E. D. Scovil and E. O. Schulz-DuBois, Phys. Rev. Lett. 2, 262 (1959).

[15] R. Alicki, J. Phys A: Math.Gen. 12, L103 (1979).

[16] R. Kosloff, J. Chem. Phys. 80, 1625 (1984).

[17] T. Feldmann and R. Kosloff, Phys. Rev. E 61, 4774 (2000).

[18] Y. Rezek and R. Kosloff, New J. Phys. 8, 83 (2006).

[19] R. Kosloff and A. Levy, Annual Review of Physical Chemistry 65, 365 (2014).

[20] U. Harbola, S. Rahav, and S. Mukamel, Euro. Phys. Lett. 99, 50005 (2012).

[21] A. E. Allahverdyan, K. Hovhannisyan, and G. Mahler, Phys. Rev. E 81, 051129 (2010).

[22] N. Linden, S. Popescu, and P. Skrzypczyk, Phys. Rev. Lett. 105, 130401 (2010).

[23] M. J. Henrich, F. Rempp, G. Mahler, Eur. Phys. J. 151, 157 (2005).

[24] P. Skrzypczyk, A. J. Short, and S. Popescu, Nature communications 5, 4185 (2014).

[25] D. Gelbwaser-Klimovsky, R. Alicki, and G. Kurizki, EPL (Europhysics Letters) 103, 60005 (2013).

[26] M. Kolář, D. Gelbwaser-Klimovsky, R. Alicki, and G. Kurizki, Phys. Rev. Lett. 109, 090601 (2012).

[27] R. Alicki, Open Syst. Inf. Dyn 21, 1440002 (2014).

[28] H. Quan, Y. x. Liu, C. Sun, and F. Nori, Physical Review E 76, 031105 (2007).

[29] J. Roßnagel, O. Abah, F. Schmidt-Kaler, K. Singer, and E. Lutz, Phys. Rev. Lett. 112, 030602 (2014).

[30] F. Binder, S. Vinjanampathy, K. Modi, and J. Goold, Physical Review E 91, 032119 (2015).

[31] L. A. Correa, J. P. Palao, D. Alonso, and G. Adesso, Scientific reports 4, 3949 (2014).

[32] R. Dorner, S. Clark, L. Heaney, R. Fazio, J. Goold, and V. Vedral, Phys. Rev. Lett. 110, 230601 (2013).

[33] L. A. Correa, J. P. Palao, G. Adesso, and D. Alonso, Physical Review E 87, 042131 (2013).

[34] R. Dorner, J. Goold, C. Cormick, M. Paternostro, and V. Vedral, Phys. Rev. Lett. 109, 160601 (2012).

[35] A. del Campo, J. Goold, and M. Paternostro, Scientific reports 4, 6208 (2014).

[36] A. S. Malabarba, A. J. Short, and P. Kammerlander, New Journal of Physics 17, 045027 (2015).

[37] D. Gelbwaser-Klimovsky, W. Niedenzu, and G. Kurizki (Academic Press, 2015) p. 329.

[38] R. S. Whitney, Physical review letters 112, 130601 (2014).

[39] A. E. Allahverdyan, K. Hovhannisyan, and G. Mahler, Phys. Rev. E 81, 051129 (2010).

[40] L. A. Correa, J. P. Palao, and D. Alonso, Physical Review E 92, 032136 (2015).

[41] K. Brandner, M. Bauer, M. T. Schmid, and U. Seifert, New Journal of Physics 17, 065006 (2015).

[42] W. S. Bakr, P. M. Preiss, M. E. Tai, R. Ma, J. Simon, and M. Greiner, Nature 480, 500 (2011).

[43] P. O. Boykin, T. Mor, V. Roychowdhury, F. Vatan, and R. Vrijen, Proceedings of the National Academy of Sciences 99, 3388 (2002).

[44] G. Bulnes Cuetara, A. Engels, and M. Esposito, New Journal of Physics 17, 055002 (2015).

[45] M. Perarnau-Llobet, K. V. Hovhannisyan, M. Huber, P. Skrzypczyk, N. Brunner, and A. Acín, Phys. Rev.
X 5, 041011 (2015).

[46] F. Rempp, M. Michel and G. Mahler, Phys. Rev. A 76, 032325 (2007).

[47] D. Segal and A. Nitzan, Phys. Rev. E 73, 026109 (2006).

[48] L. J. Schulman, T. Mor, and Y. Weinstein, Physical review letters 94, 120501 (2005).

[49] P. Oscar Boykin, Tal Mor, Vwani Roychowdhury, Farrokh Vatan, and Rutger Vrijen, Proc. Nat. Acad. Sci. 99, 3388 (2002).

[50] P. Liuzzo-Scorpo, L. A. Correa, R. Schmidt, and G. Adesso, Entropy 18, 48 (2016).

[51] P. Skrzypczyk, N. Brunner, N. Linden, and S. Popescu, Journal of Physics A: Mathematical and Theoretical 44, 492002 (2011).

[52] P. Kammerlander and J. Anders, Scientific Reports 6, 22174 (2016).

[53] M. T. Mitchison, M. P. Woods, J. Prior, and M. Huber, New Journal of Physics 17, 115013 (2015).

[54] R. Uzdin, A. Levy, and R. Kosloff, Phys. Rev. X 5, 031044 (2015).

[55] In contrast to quantum information, in quantum thermodynamics the energy basis is a preferred basis. This is due to the fact that quantum thermodynamics has a preferred set of observables e.g. heat, work and internal energy.

[56] R. Uzdin, A. Levy, and R. Kosloff, Entropy 18, 124 (2016).

[57] S. Rahav, U. Harbola, and S. Mukamel, Phys. Rev. A 86, 043843 (2012).

[58] H. P. Goswami and U. Harbola, Physical Review A 88, 013842 (2013).

[59] D. Gelbwaser-Klimovsky, W. Niedenzu, P. Brumer, and G. Kurizki, arXiv preprint arXiv:1411.1388 (2014).

[60] J. Åberg, Physical review letters 113, 150402 (2014).

[61] M. Lostaglio, D. Jennings, and T. Rudolph, Nature communications 6, 6383 (2015).

[62] M. Lostaglio, K. Korzekwa, D. Jennings, and T. Rudolph, Phys. Rev. X 5, 021001 (2015).

[63] K. Korzekwa, M. Lostaglio, J. Oppenheim, and D. Jennings, arXiv preprint arXiv:1506.07875 (2015).

[64] A. E. Allahverdyan, R. S. Gracia, T. M. Nieuwenhuizen, Phys. Rev. A 71, 046106 (2005).

[65] M. Campisi and R. Fazio, arXiv preprint arXiv:1510.06183 (2015).

[66] J. Jaramillo, M. Beau, and A. del Campo, arXiv preprint arXiv:1510.04633 (2015).

[67] A. Ü. Hardal and Ö. E. Müstecaplığlu, Scientific reports 5, 12953 (2015).

[68] F. Altintas, A. Ü. Hardal, and Ö. E. Müstecaplıŏlu, Physical Review A 91, 023816 (2015).

[69] R. Kosloff, Entropy 15, 2100 (2013).

[70] J. Goold, M. Huber, A. Riera, L. del Rio, and P. Skrzypczyk, Journal of Physics A: Mathematical and Theoretical 49, 143001 (2016)

[71] S. Vinjanampathy and J. Anders, ArXiv: 1508.06099.

[72] J. Millen and A. Xuereb, New Journal of Physics 18, $011002(2016)$

[73] R. Uzdin and R. Kosloff, New Journal of Physics 16, 095003 (2014).

[74] Eitan Geva and Ronnie Kosloff, Phys. Rev. E 49, 3903 (1994).

[75] M. O. Scully, S.-Y. Zhu, and A. Gavrielides, Physical review letters 62, 2813 (1989). 
[76] R. Alicki and M. Fannes, Phys. Rev. E 87, 042123 (2013).

[77] K. V. Hovhannisyan, M. Perarnau-Llobet, M. Huber, and A. Acín, Phys. Rev. Lett. 111, 240401 (2013).

[78] H.-P. Breuer and F. Petruccione, Open quantum systems (Oxford university press, 2002).

[79] A. E. Allahverdyan, R. Balian, and Th. M. Nieuwenhuizen, Euro. Phys. Lett. 67, 565 (2004).

[80] W. Pusz and S. Wornwicz, Commun. Math. Phys. 58, 273 (1978).

[81] A. Lenard, Journal of Statistical Physics 19, 575 (1978).

[82] R. Uzdin, arXiv preprint arXiv:1509.06289 (2015).

[83] There are limits because of the positivity constraint of the density matrix.

[84] M. O. Scully, M. S. Zubairy, G. S. Agarwal, H. Walther , Science 299, 862 (2003).
[85] M. O. Scully and M. S. Zubairy, Quantum optics (Cambridge university press, 1997).

[86] J. Roßnagel, O. Abah, F. Schmidt-Kaler, K. Singer, and E. Lutz, Physical review letters 112, 030602 (2014).

[87] G. Manzano, F. Galve, R. Zambrini, and J. M. Parrondo, arXiv preprint arXiv:1512.07881 (2015).

[88] V. Vedral, Rev. Mod. Phys. 74, 197 (2002).

[89] T. Baumgratz, M. Cramer, and M. Plenio, Physical review letters 113, 140401 (2014).

[90] M. P. Woods, N. Ng, and S. Wehner, arXiv preprint arXiv:1506.02322 (2015).

[91] As explained earlier when the machines do not interact but still work with the baths temperature used for the collective machine, then the units in the lower hemisphere no longer operate as an engine. 